\title{
Nuevos registros del género Scutellinia (Pyronemataceae, Pezizales) para la micobiota mexicana
}

\section{New records of the genus Scutellinia (Pyronemataceae, Pezizales) for the Mexican mycobiota}

\author{
Sigfrido Sierra', , Laura Izquierdo-San Agustín', Sandra Castro-Santiuste², Ibeth Rodríguez-Gutiérrez³ , Amanda \\ Alcántara-Mojica ${ }^{4}$, Lilia Pérez-Ramírez ${ }^{5}$ y Joaquín Cifuentes ${ }^{5,6}$
}

1 Universidad Nacional Autónoma de México, Facultad de Ciencias, Departamento de Biología Comparada, Laboratorio de Taxonomía de Hongos Tremeloides (Heterobasidiomycetes), Ciudad Universitaria, Av. Universidad 3000, Coyoacán, 04510 Ciudad de México, México.

2 Universidad Nacional Autónoma de México, Facultad de Ciencias, Departamento de Biología Evolutiva, Laboratorio de Biogeografía y Sistemática, Ciudad Universitaria, Av. Universidad 3000, Coyoacán, 04510 Ciudad de México, México.

3 Tecnológico de Estudios Superiores de Huixquilucan, La Magdalena Chichicaspa, 52773 Huixquilucan Estado de México, México.

4 Universidad Nacional Autónoma de México, Facultad de Medicina, Departamento de Microbiología y Parasitología, Laboratorio de Micología Médica Molecular, Ciudad Universitaria, Av. Universidad 3000, Coyoacán, O4510 Ciudad de México, México.

5 Universidad Nacional Autónoma de México, Facultad de Ciencias, Departamento de Biología Comparada, Herbario FCME Sección de Hongos, Ciudad Universitaria, Av. Universidad 3000, Coyoacán, 04510 Ciudad de México, México.

6 Universidad Nacional Autónoma de México, Facultad de Ciencias, Departamento de Biología Comparada, Laboratorio de Biodiversidad y Taxonomía de Hongos, Ciudad Universitaria, Av. Universidad 3000, Coyoacán, O4510 Ciudad de México, México.

7 Autor para la correspondencia: sigfridosg@ciencias.unam.mx

Citar como:

Sierra, S., L. Izquierdo-San Agustín, S. Castro-Santiuste, I. Rodríguez-Gutiérrez, A. Alcántara-Mojica, L. Pérez-Ramírez y J. Cifuentes. 2016. Nuevos registros del género Scutellinia (Pyronemataeae, Pezizales) para la micobiota mexicana. Acta Botanica Mexicana 79-89.

Recibido: 24 de noviembre de 2014. Revisado: 10 de septiembre de 2015. Aceptado: 13 de septiembre de 2016.
Resumen:

Antecedentes y Objetivos: El género Scutellinia es cosmopolita. Siete especies han sido reportadas en México. Se presenta una revisión taxonómica parcial del género para el país.

Métodos: Se estudiaron siete especies, siguiendo las técnicas tradicionales en micología.

Resultados clave: Tres especies se citan por primera vez en el país: Scutellinia balansae, S. colensoi y $S$. pennsylvanica. Las principales características usadas para la identificación de las especies en este estudio son los pelos marginales y la ornamentación de las ascosporas. Se realizan las descripciones taxonómicas de los tres nuevos registros y de las especies ya reportadas para el país.

Conclusiones: El conocimiento taxonómico del género Scutellinia en México sigue siendo escaso, por lo que es necesaria la elaboración de más estudios enfocados a la sistemática fúngica en el país.

Palabras clave: Fungi, ornamentación esporal, Pezizomycetidae, taxonomía.

\section{ABSTRACT:}

Background and Aims: The genus Scutellinia is cosmopolitan. Seven species have been reported in Mexico. A partial taxonomic revision of the genus Scutellinia in the country is presented.

Methods: Seven species were studied, using traditional mycological techniques.

Key results: Three species are cited for the first time in the country: Scutellinia balansae, S. colensoi and S. pennsylvanica. The main characteristics used for species identification in this study are marginal hairs and the ascospore ornamentation. Taxonomic descriptions of the three new records and previously reported species for the country are realized.

Conclusions: The taxonomic knowledge of Scutellinia in Mexico remains scarce, so the development of more studies focused on the fungal systematic in the country is necessary.

Keywords: Fungi, Pezizomycetidae, spore ornamentation, taxonomy.

\section{INTRODUCCIÓN}

El género Scutellinia (Cooke) Lambotte es cosmopolita. Se le reconoce a simple vista por su tamaño pequeño en forma de disco, con colores brillantes y por la presencia de pelos en el margen. Se sabe que es uno de los hongos que forma sus apotecios después de un incendio forestal (Lindhe et al., 2004).

Kirk et al. (2008) ubican taxonómicamente al género Scutellinia de la siguiente manera: Filum Ascomycota, Subfilum Pezizomycotina, Clase Pezizomycetes, Subclase Pezizomycetidae, Orden Pezizales, Familia Pyronemataceae. En el trabajo monográfico del género Scutellinia realizado por Schumacher (1990), se reconocieron 44 especies clasificadas en dos subgéneros: Scutellinia y Legalia T. Schumach.

En la Figura 1 se presentan las especies pertenecientes a los dos subgéneros, en la que además se adicionan 15 especies reportadas posteriormente al 
trabajo de Schumacher (1990). La monofilia del género fue demostrada por Perry et al. (2007). Choi et al. (2012) realizaron un estudio taxonómico del género en Corea.

En la bibliografía consultada (Denison, 1959; Vázquez del Mercado, 1977; Welden et al., 1979; Frutis y Guzmán, 1983; Chacón y Guzmán, 1984; Guzmán-Dávalos y Trujillo-Flores, 1984; Portugal et al., 1985; Bautista et al., 1986; Medel et al., 1988; TéllezBañuelos et al., 1988; Pompa-González y Cifuentes, 1991; Esqueda-Valle et al., 1992; Montañez, 1999; Valenzuela et al., 2004; Chanona-Gómez et al., 2007; Izquierdo-San Agustín, 2008; Izquierdo-San Agustín et al., 2013; Raymundo et al., 2013) se han reportado siete especies para México: Scutellinia asperrima (Seaver) Le Gal (nombre aceptado = Scutellinia blumenaviensis (Henn.) Le Gal), S. crinita (Bull.) Lambotte, $S$. cubensis (Berk. \& M. A. Curt) Seaver, S. setosa (Nees: Fr.) O. Kuntze, S. scutellata (L.: Fr.) Lamb., S. ulloae L. Izquierdo, S. Sierra, Rodr.-Gut., C.-Santiuste \& Cifuentes y $S$. umbrarum (Fr.) Lambotte (nombre aceptado $=S$. umbrorum (Fr.) Lambotte). Sin embargo, de estas siete especies, solo permanecen vigentes Scutellinia asperrima, S. cubensis, S. scutellata y S. ulloae. El objetivo de esta investigación, además de obtener un listado de especies y sus descripciones taxonómicas, es ampliar el conocimiento de la distribución del género Scutellinia en México.

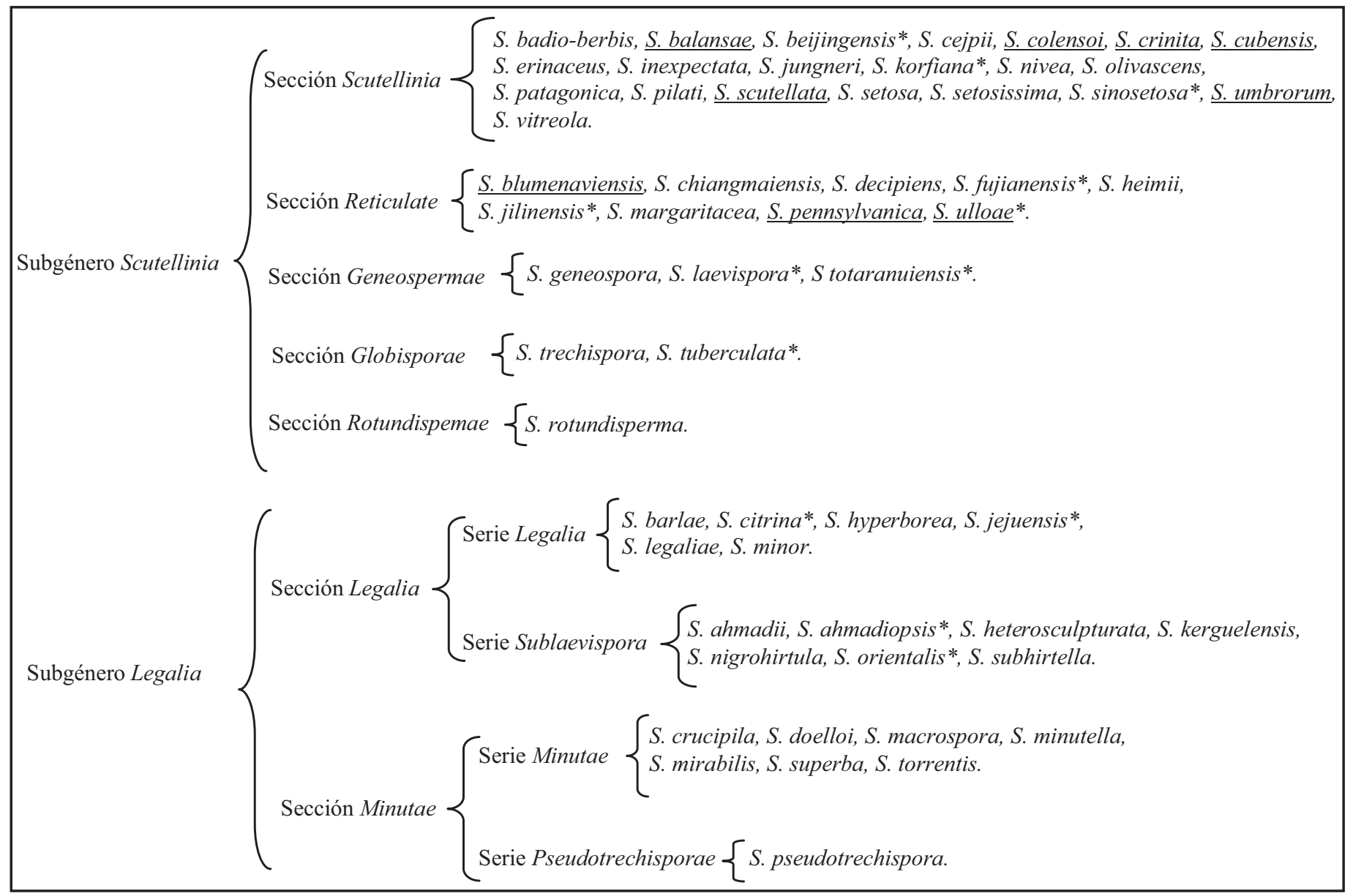

Figura 1: Especies del género Scutellinia (Cooke) Lambotte pertenecientes a los dos subgéneros. Las 13 especies marcadas con * son descritas posteriormente a Schumacher (1990). Scutellinia alleghenensis (Denison) J. Moravec y S. sinensis M.H. Liu no se pudieron ubicar en el cuadro por no tener las descripciones completas. Las especies subrayadas están citadas en México. 


\section{MATERIALES Y MÉTODOS}

Se consultó la base curatorial de Cifuentes y cols., de la sección de hongos del Herbario de la Facultad de Ciencias de la Universidad Nacional Autónoma de México (FCME), obteniéndose un listado de 70 ejemplares. Solo se consultó esta colección debido a que la mayoría de los ejemplares depositados en ésta presentan descripciones completas en fresco así como fotografías.

La mayoría de los ejemplares fueron recolectados siguiendo las técnicas propuestas por Cifuentes et al. (1986), por el tipo de información que presentan las etiquetas de descripción. Los colores fueron tomados con la guía de colores Methuen (Kornerup y Wanscher, 1978). Para el estudio micromorfológico se realizaron cortes a mano con una navaja fina, tratando de que el corte incluyera pelos del margen de los apotecios. Las observaciones se realizaron utilizando agua como medio de montaje y azul de algodón en ácido láctico. Se midieron en promedio 20 ascosporas por ejemplar, incluida la ornamentación y otra réplica de medida excluyendo ésta. Se tomaron fotografías de las ascosporas de las especies estudiadas con el microscopio electrónico de barrido (MEB), de acuerdo con la técnica descrita por Zhuang (2005). Se utilizó un microscopio Jeol (JSM-5310LV Delton-Desk-2, Tokio, Japón). Las especies fueron identificadas siguiendo los trabajos de Schumacher (1990) y Choi et al. (2012).

Debido al tamaño pequeño de los ejemplares solo algunos materiales fueron seleccionados para ser observados al MEB, que es una herramienta importante para describir la ornamentación de las esporas, la cual es determinante en la identificación. Los pelos fueron tomados del margen de los apotecios, ya que el tamaño de éstos son un carácter delimitador de las especies. Las diferencias estructurales entre los pelos del margen y los del receptáculo son muy importantes para ubicar los especímenes en los subgéneros Legalia o Scutellinia. La ornamentación de las ascosporas puede observarse en el microscopio óptico y con el microscopio de contraste por interferencia diferencial, pero no se aprecian los detalles de la misma como por ejemplo si las crestas se unen o no. Además, difícilmente puede medirse el tamaño de las verrugas y/o tubérculos. Otras veces la ornamentación no se observa porque el contenido de la espora no lo permite, o porque ésta es tan fina que no es posible observarla claramente. Las imágenes obtenidas con MEB hacen posible comparar tanto descriptiva como visualmente la ornamentación de las ascosporas. En Scutellinia la coloración de los apotecios no es tan importante, ya que el color está dado por pigmentos carotenoides que se activan dependiendo de la cantidad de luz que reciben (Schrantz y Lemoine, 1995), por lo cual no se considera relevante para la determinación de las especies pero sí para tener una descripción completa. En contraste los pelos sí son determinantes para la separación de especies, por lo que se recomienda tener cuidado en la recolecta de ejemplares para no fragmentarlos.

\section{Resultados}

De los 70 ejemplares estudiados, sólo se analizaron 50, ya que eran los que se encontraban en buen estado de conservación y sus etiquetas de recolecta presentaban datos en fresco de calidad (coloración, forma y tamaño, sustrato, entre otros). En total se determinaron siete especies: Scutellinia balansae (Speg.) Gamundí; S. colensoi Masée ex Le Gal.; S. pennsylvanica (Seaver) Denison; S. scutellata (L.) Lambotte; S. ulloae L. Izquierdo, S. Sierra, Rodr.Gut., C.-Santiuste \& Cifuentes; $S$. aff. heterosculpturata Kullman et Raitv. y $S$. aff. setosissima Le Gal, de las cuales las tres primeras son nuevos registros para México.

Se presenta una clave dicotómica para las nueve especies presentes en México: $S$. balansae, S. blumenaviensis, S. crinita, S. colensoi, S. cubensis, S. pennsylvanica, S. scutellata, $S$. ulloae y $S$. umbrorum.

\section{Clave para las especies descritas en México}

1a. Ascosporas con ornamentación reticulada ............... 2

1b. Ascosporas con ornamentación de otra forma ........... 3

2a. Retículo bien definido, crestas delgadas de hasta $2 \mu \mathrm{m}$ de alto S. blumenaviensis

2b. Retículo con crestas gruesas de 0.5-1.5 $\mu \mathrm{m}$ de alto ..... S. pennsylvanica

3a. Ornamentación tuberculada; tubérculos de cilíndricos a obpiriformes S. ulloae 
3b. Ornamentación verrucosa, pústulo-crestada a tuberculada; nunca obpiriformes .................................... 4

4a. Ornamentación verrucosa ........................................ 5

4b. Ornamentación tuberculada a pústulo-crestada ........ 6

5a. Ornamentación con verrugas pequeñas $(<1 \mu \mathrm{m}$ de largo y alto) S. crinita

$5 b$. Ornamentación con verrugas grandes $(>1 \mu \mathrm{m}$ de largo y alto) S. scutellata

6a. Ornamentación tuberculada S. umbrorum

6b. Ornamentación pústulo-crestada 7

7a. Pelos del margen generalmente $<1000 \mu \mathrm{m}$ S. colensoi

7b. Pelos del margen generalmente $>1000 \mu \mathrm{m}$............... 8 8a. Ascas de $170-200 \times 11.5-15 \mu \mathrm{m}$ S. balansae

8 b. Ascas de 250-270 × 16-20 $\mu \mathrm{m}$ S. cubensis

Scutellinia balansae (Speg.) Gamundí, Deutsche Zeitschrift für Chirurgie 1(2): 85. 1956. Fig. 2A, H.

Apotecio de 1-22 mm de diámetro, discoide plano, margen levantado y ondulado, himenio de color naranja persa (6A7) a naranja mandarina (6B8), superficie externa de color naranja persa (8A8); pelos del margen de (648-)1118-1176 × 20.5-40.6 $\mu \mathrm{m}$, pared de 2.5-4.5 $\mu \mathrm{m}$ de ancho, multiseptados, ocasionalmente bifurcados en el ápice, base de simple a multifurcada de color pardo oscuro a negro; ascas de 170-200 × 11.5-15 $\mu \mathrm{m}$, cilíndricas, octosporadas; ascosporas de (15-)16.4-18.5(-19) × (9-)10-13 $\mu$ m, elipsoidales, de borde irregular, con una a dos gútulas, algunas presentando burbujas de De Bary, ornamentación formada por verrugas truncas, de forma irregular, creando un borde caprichoso, al centro las verrugas se ven redondeadas, aisladas, aunque algunas llegan a unirse formando pequeñas crestas, de 0.3$0.9(-1.9) \mu \mathrm{m}$ de alto $\times 0.2-1.6(-2.8) \mu \mathrm{m}$ de ancho correspondiendo a una ornamentación tipo pústulo-crestada; paráfisis filiformes, clavadas en el ápice, septadas, sobrepasando el nivel de las ascas, de 2-5.5(-6.24) $\mu \mathrm{m}$ de ancho.

Hábitat: lignícola; en bosque mesófilo de montaña y cafetales; de 1450 a 2400 m s.n.m.; se colectó en los meses de septiembre a octubre.
Material revisado: MÉXICO. Chiapas, municipio de Cacahoatán, ejido Mixcum, 18.X.1997, J. Cifuentes 4021 (FCME 9438). Hidalgo, municipio de Tlanchinol, $60 \mathrm{~km}$ adelante de Molango, carr. Pachuca - Tampico, 14.IX.1993, S. Sierra 182 (FCME 6219). Municipio de Omitlán de Juárez, km 18 carr. Pachuca - Tampico, 02.X.1989, M. Villegas-Ríos 1308 (FCME 2675).

Observaciones: En el material de Hidalgo la altura de las verrugas llega hasta $0.9 \mu \mathrm{m}$, mientras que en el ejemplar de Chiapas va de 0.9 a $1.9 \mu \mathrm{m}$. Sin embargo, coinciden en la forma de la ornamentación. Por otro lado, aunque en los ejemplares estudiados algunas verrugas son más angulares, la pared de los pelos más delgada y las esporas más pequeñas, son similares a la especie descrita por Schumacher (1990). De acuerdo con este autor, $S$. balansae tiene ascosporas de 18.2-24.8 $\times 12.2-14.8 \mu \mathrm{m}$, presenta una ornamentación de verrugas redondeadas de diferente tamaño que miden hasta $1.6 \mu \mathrm{m}$ de alto $\times 0.6$ $1.5 \mu \mathrm{m}$ de ancho, dos o tres verrugas pueden fusionarse de forma irregular, con apariencia ameboide, interespaciadas de forma irregular; pelos de 150-1110 $\times 24-54 \mu \mathrm{m}$, pared de 4.5-8.4 $\mu \mathrm{m}$ de ancho. Coinciden en el resto de las características como el sustrato, aunque $S$. balansae descrita por Schumacher (1990) posee un apotecio (4-12 $\mathrm{mm}$ de diámetro) y paráfisis pequeñas (2.3-3.5 $\mu \mathrm{m}$ de ancho). La especie se distribuye en Argentina, Burundi, Costa Rica, Cuba, Paraguay, Trinidad, Uganda, Zaire y Zimbabwe (Schumacher, 1990). En este trabajo se considera un nuevo registro para México.

Scutellinia colensoi Masée ex Le Gal., Bulletin trimestriel de la Societé Mycologique de France 83: 356. 1967.

Fig. 2B.

Apotecio de 3-10 mm de diámetro, discoide cóncavo, margen ondulado a extendido en los ejemplares más grandes, himenio de color naranja pardusco (6B8) a cobre (7C8), superficie externa de color siena quemado (7D8); pelos del margen de (240-)570-1000(-1200) $\mu \mathrm{m} \times 30-51$ $\mu \mathrm{m}$, pared de 5-7.7 $\mu \mathrm{m}$, flexibles, multiseptados, con base 

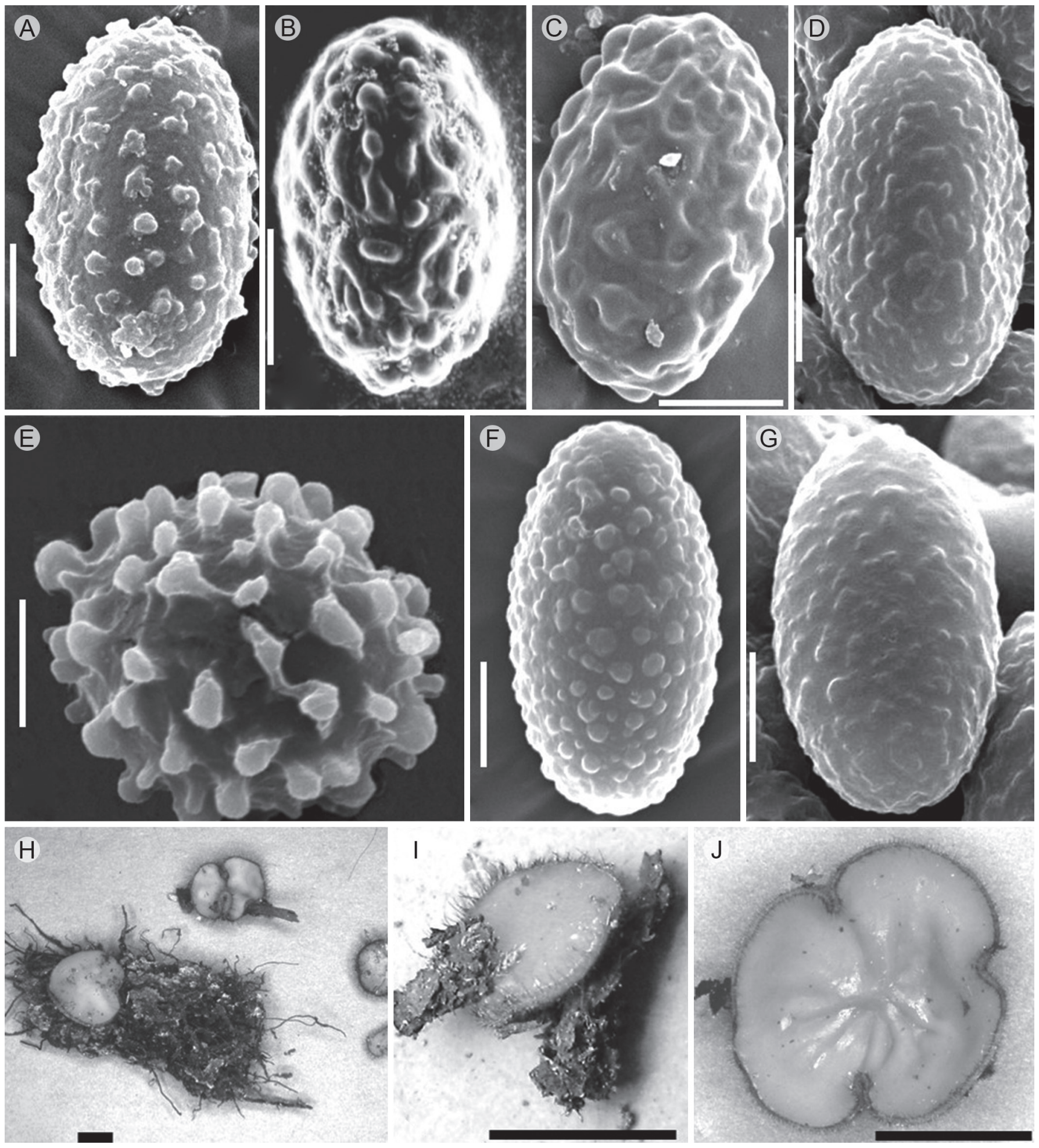

Figura 2: A-G: Ascosporas en MEB. A. Scutellinia balansae (Speg.) Gamundí; B. S. colensoi Massee ex Le Gal; C. S. pennsylvanica (Seaver) Denison; D. S. scutellata (L.) Lambotte; E. S. ulloae L. Izquierdo; F. S. aff. heterosculpturata Kullman \& Raitv.; G. S. aff. setosissima Le Gal. H-J: Ascomas en fresco. H. S. balansae; I. S. colensoi; J. S. pennsylvanica. Barras de referencia: A-G=5 $\mu \mathrm{m}$. H-J=10 mm. 
simple a bifurcada; ascas de 201.6-228 $\times 14.4 \mu \mathrm{m}$, octosporadas, cilíndricas; ascosporas de (15.6-)17.7-19(-21) $\times$ (9.4-)10.4-12(-12.5) $\mu \mathrm{m}$, elipsoidales, ornamentación de tubérculos redondeados, ocasionalmente aislados hacia el borde, pero la mayoría se unen formando costillas de 0.4-1.6 $\mu \mathrm{m}$ de alto y de 1.2-2.3 $\mu \mathrm{m}$ de largo, también hay pequeñas líneas sinuadas entre los tubérculos que corresponden a un tipo de ornamentación pústulo-crestada; paráfisis filiformes de hasta $5 \mu \mathrm{m}$ de ancho, septadas, alargadas en el ápice.

Hábitat: terrícola, lignícola, un ejemplar está asociado con musgo; en bosque mesófilo de montaña; de 2200 a 2250 m s.n.m.; se colectó en el mes de agosto.

Material revisado: MÉXICO. Estado de México, municipio de Temascaltepec, desviación a El Polvorín, km 54 carr. Toluca - Temascaltepec; 25.VIII.1989; J. Cifuentes 2922 (FCME 3739), 2921 (FCME 3741).

Observaciones: Esta especie se caracteriza por el tamaño del apotecio (2-6 mm de diámetro), de los pelos de $(240-1000(-1200) \times 25-50 \mu \mathrm{m})$, grosor de la pared (5-7 $\mu \mathrm{m})$, tamaño de las ascosporas de (17.2-20.3 $\times$ 9.8$11.6 \mu \mathrm{m})$ y por la ornamentación de verrugas irregulares ameboideas, de $1.5 \mu \mathrm{m}$ de alto $\times 2.2 \mu \mathrm{m}$ de ancho, que frecuentemente se unen para formar crestas irregulares y cortas, interconectadas para formar un seudo-retículo; en la superficie de la pared se observan líneas sinuosas entre las verrugas adyacentes, que pueden elevarse para formar una red en forma de telaraña debajo de la ornamentación ya mencionada (Schumacher, 1990). Las características mencionadas coinciden con las de los ejemplares estudiados, por lo que no hay duda que se trata de $S$. colensoi. Otros caracteres que coinciden son el hábitat (sobre musgo), el tamaño del apotecio y las paráfisis (forma y tamaño). La especie se distribuye en Australia, Brasil, Cuba, ex-URSS, Japón, Nueva Guinea, Nueva Zelanda, República Checa, Slovaquia, Sudáfrica, Tailandia, Tanzania y Zimbabwe (Schumacher, 1990). Es un nuevo registro para México.
Scutellinia pennsylvanica (Seaver) Denison, Mycologia 51: 619. 1959. Fig. 2C, J.

Apotecio de 2-20 mm de diámetro, en forma de disco, algunos ligeramente cóncavos, margen convoluto, carnoso (quebradizo) a cartilaginoso, himenio de color amarillo oro (Methuen 5B7) a rojo naranja (8A8), superficie externa blanquecino naranja (5A2) a rojo naranja (8A8); pelos del margen de 432-1905.6 × 20-72(-80) $\mu \mathrm{m}$, pared de 2.5$11.4 \mu \mathrm{m}$, ensanchándose hacia la base, simples a multifurcados, multiseptados, de color café oscuro a negro; ascas de 134.9-260 × 8.3-17.5 $\mu \mathrm{m}$, cilíndricas, octosporadas; ascosporas de (15.3-)16.5-22(-23.9) × (9.3-)10-13(-13.5) $\mu \mathrm{m}$, elipsoidales con borde irregular, gutuladas, algunas con burbujas de De Bary, la ornamentación vista en el microscopio óptico consiste en verrugas redondeadas que se unen por líneas delgadas o anchas (de modo que no se distingue un tubérculo de otro), en MEB se observa un retículo de verrugas redondeadas que se unen formando crestas o líneas sinuadas de 0.5-1.5 $\mu \mathrm{m}$ de alto, hacia los polos, algunas verrugas llegan a verse aisladas, retículo uniforme y espaciado, a veces éste es más abundante, corresponde a una ornamentación de tipo tubérculo-reticulado; paráfisis filiformes, clavadas en el ápice, delgadas, septadas, sobrepasando las ascas hasta $5.47 \mu \mathrm{m}$.

Hábitat: terrícola, lignícola, algunas veces asociado con musgo; crece en bosques de Abies, Abies-Pinus, Pinus-Quercus, Quercus y bosque mixto; de 2150 a 2900 m s.n.m.; se colectó en los meses de julio a octubre.

Material revisado: MÉXICO. Chihuahua, municipio de Bocoyna, La Laguna, $5 \mathrm{~km}$ al Este de Bocoyna, 20.IX.1992, A. Moreno Fuentes IX-9 (FCME 5636). Estado de México, municipio de Amanalco, Agua Bendita, km 39 carr. Toluca - Amanalco - Valle de Bravo, 07.VII.1990, M. Villegas-Ríos 1360 (FCME 3200); 1359 (FCME 3207). Municipio de Ocuilan de Arteaga, Laguna de Quila, 21.IX.1995, M. Aguilar s.n. (FCME 7167). Guanajuato, municipio de Guanajuato, Cañada de Llano Largo, Sierra de Santa Rosa, 
26.VIII.1995, J. Guerrero y J. L. Villarruel-Ordaz 389 (FCME 7720); 09.VIII.1995, M.C. Rojas-García et al. 2061 (FCME 7727); R. Amaya-Luna et al. 2062 (FCME 7730); 10.IX.1995, E. Galván-Mejía 05 (FCME 7738). Guerrero, municipio Chilpancingo de los Bravo, Cañada de Agua Fría, Omiltemi, 13.VIII.1984, L.C. Luna García s.n. (FCME 12777). Michoacán, municipio de Angangueo, Llano Villalobos, Reserva de la Biósfera Mariposa Monarca, 28.VIII.1998, E. Pellicer-González 54 (FCME 15014); 12.IX.1998, T. Pérez Corona y J.L. Villarruel-Ordaz 651 (FCME 15044). Municipio de Ocampo, Laguna verde, cerca de Ocampo; 23.IX.2000, J. Cifuentes 2000-345 (FCME 17636). Querétaro, municipio de Amealco, km 11, desviación a Laguna de Servín, 01.X.2001, G. Vidal-Gaona 330 (FCME 18479). Tlaxcala, municipio de Tlaxco, Parque recreativo El Rodeo, 04.IX.1992, G. Vidal-Gaona 60 (FCME 4808), 70 (FCME 4813), 61 (FCME 4830), 62 (FCME 4818), 65 (FCME 5014); 04.VII.1992, M. Villegas-Ríos 1472 (FCME 4810); 25.IX.1992; M. Puebla-Amado y M.A. Hernández-Muñoz 527 (FCME 5034); J. López-Salado y M.A. Hernández-Muñoz 526 (FCME 5042); km 4-5 del Rosario - Parque recreativo El Rodeo, 17.VII.1992, A. Pompa-González 193 (FCME 4997).

Observaciones: A veces el retículo de las ascosporas observado en el microscopio óptico consiste en verrugas unidas por líneas delgadas, por lo que podría llegar a confundirse con $S$. chiangmaiensis T. Schumach., que posee un retículo alveolado, de $4.5 \mu \mathrm{m}$ de alto $\times 1.5-2.7$ $\mu \mathrm{m}$ de largo. En los ejemplares estudiados los pelos son más anchos, de pared más gruesa y las verrugas ligeramente pequeñas con respecto a $S$. pennsylvanica descrita por Schumacher (1990); aunque se observa una variación en la abundancia y el espaciado de la ornamentación, hay correspondencia en las demás características macro y microscópicas. Scutellinia pennsylvanica se distribuye en Argentina, Cuba, Estados Unidos de América e India (Schumacher, 1990). Éste es el primer registro de la especie en México por lo cual se reveló que es una especie de amplia distribución.
Scutellinia scutellata (L.: Fr.) Lamb., Mémoires de la Société Royale des Sciences de Liège, Suppl. 1: 299. 1887. Fig. 2D.

Apotecio de 2-12 mm de diámetro, en forma de disco plano, margen ondulado, color del himenio naranja pálido (Methuen 6A3) a naranja rojizo (8A8), superficie externa de color salmón (6A5) a naranja grisáceo (7B4); pelos del margen de 504-1920 $\mu \mathrm{m}$ de largo $\times(10-) 31.5-52.5(80)$ $\mu \mathrm{m}$ de ancho, pared de 1.6-3.7 $\mu \mathrm{m}$ de ancho en el ápice, hasta (4.8-)7-10.8 $\mu \mathrm{m}$ de ancho aproximándose a la base; rectos, de color café parduzco (6F8) a café obscuro (7F8), rígidos, multiseptados, de simples a trifurcados, algunos presentan una protuberancia en la base, o de base ancha; ascas cilíndricas, octosporadas de (259-)272-287 × 16.36$18.4 \mu \mathrm{m}$, algunos ejemplares presentando 6 esporas por asca; ascosporas elipsoidales, de (16.4-)17.5-21.5(-22.9) $\times(9.4) 10.5-13.5(-19.8) \mu \mathrm{m}$, con una, dos o muchas gútulas, de borde liso, ornamentación consistente de verrugas irregulares de 0.3-1 $\mu \mathrm{m}$ de alto y de 0.4-2.9 $\mu \mathrm{m}$ de largo, algunas se ven aisladas, la mayoría se unen pudiendo formar placas o crestas, correspondiendo a una ornamentación tipo verrucosa; paráfisis filiformes, sobresaliendo de las ascas, clavadas en el ápice, de 6-7.3 $\mu \mathrm{m}$ de ancho.

Hábitat: lignícola, terrícola, humícola, algunas veces forma asociación con musgo y madera; en bosque de Pinus-Quercus, bosque mesófilo de montaña, bosques mixtos; de 2100 a 2710 m s.n.m.; se colectó en los meses de agosto a octubre.

Material revisado: MÉXICO. Estado de México, municipio de Amanalco, km 55.5 carr. Toluca - Amanalco - Valle de Bravo, 22.VIII.1990, J.C. Quiroz Mendoza y L. Pérez-Ramírez 1245 (FCME 3141). Municipio de Valle de Bravo, km 4 circuito Manantiales - Avándaro, 19.VIII.1989, A. Pompa-González 67 (FCME 2292); km 21 de la desviación a Valle de Bravo, carr. Toluca - Temascaltepec, 18.IX.1989, A. Pompa-González 74 (FCME 2366); 07.X.1989, A. Pompa-González 91 (FCME 2374), 92 (FCME 2387). Guanajuato, municipio de Guanajuato, 
km 9-11 de Sta. Rosa de Lima - Monte de San Nicolás, 29.IX.1995, E. Carrasco-Ramírez y L. Pérez-Ramírez 2133 (FCME 7367). Guerrero, municipio de Chichihualco, $\mathrm{km} 4.5$ entre el carrizal y Atoyac, 30.VIII.1980, V. Trejo 17 (FCME 10292); municipio de Taxco, Parque Cerro del Huizteco, 05.VII.1986, F. López-Monter y M. VillegasRíos 727 (FCME 5620); km 8-10 carr. Taxco - Tetipac, 26.VII.1986, R. Gutiérrez N. 1027 (FCME 5603). Michoacán, municipio de Ciudad Hidalgo, Mil Cumbres, km 182, carr. Toluca - Morelia, 01.IX.1994, E. Méndez-Cardiel 61 (FCME 7039). Municipio de Ocampo, Laguna Verde, cerca de Ocampo, 19.VIII.2000, R. Vázquez y S. Sierra 809 (FCME 17392). Nayarit, municipio de Tepic, La Noria, Reserva Ecológica del Cerro San Juan, 28.VIII.1991, E. Soria-Castro y M.A. Hernández-Muñoz 256 (FCME 4123); 13.IX.1992, M. Villegas-Ríos 1521 (FCME 5333). Tlaxcala, municipio de Tlaxco, a $5 \mathrm{~km}$ de El Rosario, 07.IX.1992, A. Pompa-González 157 (FCME 23264), 162 (FCME 23265), 163 (FCME 23266), parque recreativo El Rodeo, 04.IX.1992, G. Vidal-Gaona 58 (FCME 4750), 68 (FCME 4817), km 4-5 del Rosario - Parque recreativo El Rodeo, 26.IX.1992, A. Pompa-González 207 (FCME 5501).

Observaciones: El tamaño de los pelos y esporas son caracteres diagnósticos de $S$. scutellata (Schumacher, 1990). Existe afinidad entre $S$. scutellata y $S$. crinita citadas por Schumacher (1990) por lo que podría confundirse. Sin embargo, en la descripción y en las fotografías de las esporas, $S$. crinita presenta una ornamentación tipo verrucosa y no forma placas, como $S$. scutellata. Los ejemplares estudiados forman placas y presentan ornamentación escasa, por lo que se adscriben a $S$. scutellata. Es una especie de amplia distribución a nivel mundial. En México ya había sido registrada para Chiapas, Ciudad de México, Estado de México, Hidalgo, Jalisco, Morelos, Oaxaca, Sonora y Veracruz. En este trabajo se considera un nuevo registro para los estados de Guanajuato, Michoacán, Nayarit y Tlaxcala.

Scutellinia ulloae L. Izquierdo, S. Sierra, Rodr.-Gut., C.-Santiuste \& Cifuentes, Mycotaxon 125: 258. 2013. Fig. 2E.
Apotecio sésil, discoide, de 1-4 mm de diámetro, himenio liso, de color naranja rojizo (Methuen 7A8), superficie externa blanquecina, margen recto y consistencia cartilaginosa; pelos del margen de 648-1224 $\times$ 18-35(-41) $\mu \mathrm{m}$, pared de 3-3.5 $\mu \mathrm{m}$, de color café, simples, aunque hay bifurcados, rectos, no hay diferencia entre pelos marginales y pelos laterales; ascas de 240-272 × 15.6-31.2 $\mu \mathrm{m}$, cilíndricas, operculadas, octosporadas; ascosporas subglobosas de (15-)17-17.5 $\times 12-13(-14) \mu \mathrm{m}$, una gútula a multigutuladas, la ornamentación consiste en tubérculos de 2-2.5 $\mu \mathrm{m}$ de alto $\times 2 \mu \mathrm{m}$ de ancho, los tubérculos son de cilíndricos a obpiriformes (clavados hacia el ápice y se adelgazan a la base), algunos se unen por líneas delgadas, la ornamentación se distingue fácilmente en el microscopio óptico, en la esporas teñidas con azul de algodón; paráfisis filiformes, clavadas en el ápice, delgadas, septadas, sobresalen de las ascas.

Hábitat: terrícola; en bosque de Pinus a $1950 \mathrm{~m}$ s.n.m.; se recolectó un único material en el mes de septiembre.

Material revisado: MÉXICO. Estado de México, municipio de Temascaltepec, desviación a Presa Chica, km 71 carretera Toluca - Tejupilco, 23.IX.1988, E. M. Hilario y M. Villegas 1130 (FCME 14557).

Observaciones: Siguiendo a Schumacher (1990), la especie más cercana al material estudiado es Scutellinia chiangmaiensis $\mathrm{T}$. Schumach., pero la ornamentación es muy diferente, ya que mientras en $S$. chiangmaiensis es tubérculo-reticulada, dando una apariencia alveolada, en $S$. ulloae es tuberculada, con tubérculos cilíndricos a obpiriformes, ligeramente unidos por crestas pequeñas y delgadas, sin llegar a formar un retículo. El tipo de vegetación es bosque mixto deciduo para $S$. chiangmaiensis y bosque de coníferas para $S$. ulloae. Este ejemplar fue erróneamente determinado por Pompa-González y Cifuentes (1991) como S. diaboli (Velen.) Le Gal (=S. trechispora (Berk. \& Broome) Lambotte). 
Scutellinia aff. heterosculpturata Kullman et Raitv., Folia cryptogamica Estonica 7: 4. 1977. Fig. 2F.

Apotecio de 4-10 mm de diámetro, discoide plano, con margen ondulado en los ejemplares de mayor tamaño, color naranja rojizo (8B8); pelos del margen de 864-960 × 30-50 $\mu \mathrm{m}$, pared de 2-3 $\mu \mathrm{m}$, multiseptados, ligeramente curvados, ramificación simple a trifurcada, acuminados en la punta, de color café; ascas de 152-213 × 14.6-17.7 $\mu \mathrm{m}$, cilíndricas, octosporadas; ascosporas de 18.9-20.5(-21) × (10.5-)11$12.5(-13) \mu \mathrm{m}$ fusiformes, ligeramente acuminadas en los polos, gutuladas, algunas presentando burbujas de De Bary, la ornamentación consiste en verrugas aisladas, redondeadas, homogéneamente distribuidas, de diferente tamaño, algunas llegan a fusionarse, de 0.3-0.5 $\mu \mathrm{m}$ de alto $\times 0.4$ hasta $2 \mu \mathrm{m}$ de largo, pero no forman placas; paráfisis filiformes, delgadas, septadas, de 3-4.7 $\mu \mathrm{m}$ de ancho, sobresaliendo de las ascas.

Hábitat: terrícola, se encuentra en el bosque de $P i$ cea-Pinus, de 2350 a 2450 m s.n.m., se recolectó un único material en el mes de septiembre.

Material revisado: MÉXICO. Chihuahua, municipio de Bocoyna; $1 \mathrm{~km}$ adelante de El Ranchito, camino San Juanito - Basaseachi, 14.IX.1996, L. Pérez-Ramírez 2213 (FCME 9315).

Observaciones: En la descripción de $S$. heterosculpturata de Schumacher (1990) se presentan tubérculos de 0.5-1.2 $\mu \mathrm{m}$ de ancho, con tubérculos más grandes de hasta $1.5 \mu \mathrm{m}$ de alto por $3.5 \mu \mathrm{m}$ de ancho; en el ejemplar revisado los tubérculos no son tan grandes (hasta $0.5 \mu \mathrm{m}$ de alto); hay coincidencia en cuanto al tamaño y la forma de los pelos así como la forma de los tubérculos, que hacen suponer que podría pertenecer a esta especie. Debido a que solo se estudió un ejemplar, es necesario, además de hacer más recolecciones en el área, solicitar al herbario EULS de la Estonian University of Life Sciences el material tipo para verificar si se trata de esta especie o no. Asimismo, en GenBank se encuentran secuencias de algunos ejemplares de esta especie, por lo que en un futuro se podrá comple- mentar la información morfológica con los datos moleculares para una correcta determinación del material.

Scutellinia aff. setosissima Le Gal, Bulletin trimestriel de la Societé Mycologique de France 84: 378. 1969. Fig. 2G.

Apotecio de 1-8 mm de diámetro, margen diferenciable, himenio de color naranja fuerte (6A8) a naranja rojizo (7B-C8), cartilaginoso; pelos del margen de 864-1368 $\times$ 35-45 $\mu \mathrm{m}$, pared de hasta $3 \mu \mathrm{m}$ de ancho, de base ancha, simples a bifurcados, multiseptados, de color café claro; ascas de 250-260 × 13.8-16.6 $\mu \mathrm{m}$, cilíndricas, octosporadas; ascosporas de (9-)19-20 × (5-)9.6-10(13.8) $\mu \mathrm{m}$, elipsoidales, gutuladas a multigutuladas, verrugas de forma irregular, ameboideas, algunas se unen formando pequeñas "agregaciones de verrugas" apenas visibles en el microscopio óptico, de 0.8-1 $\mu \mathrm{m}$ de largo $\times 0.5 \mu \mathrm{m}$ de alto; paráfisis filiformes, septadas, subclavadas en el ápice, de 4.2-7.8 de ancho, sobresaliendo al nivel de las ascas.

Hábitat: lignícola, creciendo en bosque de PinusQuercus, de 2490 a 2600 m s.n.m., en los meses de agosto y septiembre.

Material revisado: MÉXICO. Michoacán, Municipio de Zinapécuaro, carretera a San Pedro Jácuaro - Los Azufres, desviación al Balneario Eréndira, 30.VIII.1994, E. Méndez-Cardiel 49 (FCME 7123); 02.IX.1994, E. Méndez-Cardiel 68 (FCME 7146).

Observaciones: El material estudiado concuerda con la descripción de S. setosissima (Schumacher, 1990), principalmente por el tamaño de los pelos, pero la pared es más delgada en los ejemplares estudiados. Las ascosporas en nuestros materiales son ligeramente más pequeñas y la ornamentación más grande; por ello se designa como afín. Schumacher (1990) no describe el tamaño de la ornamentación, quizá porque la ornamentación es tan pequeña que no es susceptible de ser medida, corroborándose con sus fotografías de las ascosporas en MEB. 


\section{DISCUSIÓN}

Esta contribución constituye la primera en México en tratar este género a profundidad. La revisión bibliográfica puso en evidencia que la nomenclatura para algunas especies de Scutellinia citadas para el país es inválida o errónea. Scutellinia asperrima es sinónimo de Scutellinia blumenaviensis (Schumacher, 1990). Denison (1959) citó a S. asperrima de México, por lo que el registro actual es $S$. blumenaviensis. Scutellinia setosa se reporta en la tesis de Montañez (1999), pero aún no ha sido citada en un trabajo formal (revista científica, libro, listado, etc.) por lo que su registro todavía no es válido para México. Scutellinia umbrarum fue citado por Esqueda-Valle et al. (1992), pero es un nombre no registrado en Index Fungorum (2016) ni en MycoBank (2016). Phillips et al. (2010) citan y describen una especie de Scutellinia con el epíteto especifico de "umbrarum". Quizá Esqueda-Valle et al. (1992) se referían a $S$. umbrorum, ya que su descripción es similar a la que presenta Schumacher (1990).

Con respecto a los reportes de Scutellinia crinita (citada de Oaxaca por Raymundo et al., 2013) y $S$. cubensis (citada de Chiapas por Medel et al., 1988) siguen conservando su registro para el país. De acuerdo con lo anterior y considerando los tres nuevos registros que aquí se presentan, son nueve las especies de Scutellinia citadas hasta ahora para México.

En la Figura 1 se hace referencia a estas nueve especies, las cuales pertenecen al subgénero Scutellinia y a las secciones Scutellinia (6 especies) y Reticulate (3). En el caso de las dos especies aquí citadas como Scutellinia aff. heterosculpturata y $S$. aff. setosissima, si se llegara a confirmar la determinación de estas especies con más recolectas y estudios de MEB, serían consideradas como nuevos registros para el país. Por otra parte, en caso de no coincidir, es muy probable que se trate de dos nuevas especies para la ciencia.

Con respecto a la distribución de las especies aquí estudiadas en los diferentes tipos de vegetación, podemos observar que en los bosques templados son más abundantes. Asimismo los sustratos que predominaron en los ejemplares aquí estudiados son el humícola y lignícola. Los estados mejor representados después de este estudio son Chiapas y
Estado de México (con cuatro especies), Michoacán y Sonora (con tres), Chihuahua, Guanajuato, Guerrero, Hidalgo, Oaxaca, Tlaxcala y Veracruz (con dos) y CDMX, Jalisco Morelos, Nayarit y Querétaro con una especie cada uno. Se espera que la revisión de más herbarios y nuevas recolecciones en diferentes zonas del país aumente el número de especies de Scutellinia presentes en nuestro país. Se recomienda, para tener una certeza más exacta de las determinaciones, además de realizar observaciones al MEB, utilizar técnicas moleculares para la identificación de las especies.

\section{AgradeCIMIENTOS}

Las fotografías de MEB fueron realizadas por Silvia Espinosa Matías, Laboratorio de Microscopía de Barrido, Facultad de Ciencias, UNAM. El trabajo fue financiado por la UNAM, PAPIIT-DGAPA IN218008 e IN207311.

\section{LITERATURA CITADA}

Bautista, N., S. Chacón y G. Guzmán. 1986. Ascomicetes poco conocidos de México, III. Especies del estado de Morelos. Revista Mexicana de Micología 2: 85-104.

Chacón, S. y G. Guzmán. 1984. Nuevas observaciones sobre los hongos, líquenes y mixomicetos de Chiapas. Boletín de la Sociedad Mexicana de Micología 19: 245-252.

Chanona-Gómez, F., R. H. Andrade-Gallegos, J. CastellanosAlbores y J. E. Sánchez. 2007. Macromicetos del Parque Educativo Laguna Bélgica, municipio de Ocozocoautla de Espinosa, Chiapas, México. Revista Mexicana de Biodiversidad 78: 369-381.

Choi, Y. J., H. D. Shin, J. G. Han y D. H. Pfister. 2012. Scutellinia (Pezizales) in Korea, with a new species and eight new records. Nova Hedwigia 97(3-4): 457-476.

Cifuentes, J., M. Villegas y L. Pérez-Ramírez. 1986. Hongos. In: Lot, A. y F. Chiang (eds.). Manual de Herbario. Consejo Nacional de la Flora de México, A.C. México, D.F., México. pp. 55-64.

Denison, W. C. 1959. Some species of the genus Scutellinia. Mycologia 51: 605-635.

Esqueda-Valle, M., E. Pérez-Silva y A. M. Coronado. 1992. Nuevos registros de Pezizales para Sonora. Revista Mexicana de Micología 8: 43-54. 
Frutis, I. y G. Guzmán. 1983. Contribución al conocimiento de los hongos del estado de Hidalgo. Boletín de la Sociedad Mexicana de Micología 18: 219-265.

Guzmán-Dávalos, L. y F. Trujillo-Flores. 1984. Hongos del estado de Jalisco IV. Nuevos registros. Boletín de la Sociedad Mexicana de Micología 19: 319-326.

Index fungorum. An international project to index all formal names in the Fungi Kingdom. http://www.indexfungorum.org/ (consultado agosto de 2016).

Izquierdo-San Agustín, L. 2008. Contribución al conocimiento taxonómico del género Scutellinia en México. Tesis de licenciatura. Facultad de Ciencias, Universidad Nacional Autónoma de México. México, D.F., México. 51 pp.

Izquierdo-San Agustín, L., S. Sierra, I. Rodríguez-Gutiérrez, S. Castro-Santiuste y J. Cifuentes. 2013. A new species of Scutellinia from México. Mycotaxon 125: 257-262.

Kirk, P. M., P. F. Cannon, D. W. Minter y J. A. Stalpers. 2008. Dictionary of the Fungi. 10th ed. CAB International. Wallingford, UK. $771 \mathrm{pp}$.

Kornerup, A. y J. H. Wanscher. 1978. Methuen Handbook of Colour. Eyre, UK. 252 pp.

Lindhe, A., N. Åsenblad y H.-G. Toresson. 2004. Cut logs and high stumps of spruce, birch, aspen and oak-nine years of saproxylic fungi succession. Biological Conservation 119: 443-454.

Medel, R., S. Chacón y G. Guzmán. 1988. Ascomycetes lignícolas de México, II. Algunos Pyrenomycetes y Discomycetes. Micología Neotropical Aplicada 1: 87-96.

Montañez, A. 1999. Análisis de la diversidad de Macromicetos que crecen en bosques de encino del Municipio de Chapa de Mota, Estado de México. Tesis de licenciatura. Facultad de Ciencias, Universidad Nacional Autónoma de México. México, D.F., México. 86 pp.

Mycobank. An on-line database aimed as a service to the mycological community by documenting mycological nomenclatural novelties. http://www.mycobank.org/ (consultado agosto de 2016).

Perry, B. A., K. Hansen y D. H. Pfister. 2007. A phylogenetic overview of the family Pyronemataceae (Ascomycota, Pezizales). Mycological Research 111: 549-571.
Phillips, R., G. Kibby and N. Foy. 2010. Mushrooms and Other Fungi of North America. Firefly Books. Buffalo, USA. 384 pp.

Pompa-González, A. y J. Cifuentes. 1991. Estudio taxonómico de los Pezizales de los estados de Guerrero, Hidalgo, Estado de México y Michoacán. Revista Mexicana de Micología 7: 87-112.

Portugal, D., E. Montiel, L. López y V. M. Mora. 1985. Contribución al conocimiento de los hongos que crecen en la región del Texcal, estado de Morelos. Revista Mexicana de Micología 1: 401-412.

Raymundo, T., E. Aguirre-Acosta, S. Bautista-Hernández, M. Contreras-Pacheco, P. Garma, H. León-Avendaño y R. Valenzuela. 2013. Catálogo de los Ascomycota en los bosques de Santa Martha Latuvi, Sierra Norte, Oaxaca, México. Boletín de la Sociedad Micológica de Madrid 37: 13-29.

Schrantz, J. P. y Y. 1995. Carotenoid composition of mycelium and apothecia in the discomycete Scutellinia umbrarum. Phytochemistry 40(1): 33-35.

Schumacher, T. 1990. The genus Scutellinia (Pyronemataceae). Opera Botanica 101: 1-107.

Téllez-Bañuelos, C., L. Guzmán-Dávalos y G. Guzmán. 1988. Contribución al conocimiento de los hongos de la reserva de la Biosfera de la Sierra de Manantlán, Jalisco. Revista Mexicana de Micología 4: 123-130.

Valenzuela, R., T. Raymundo y M. R. Palacios. 2004. Macromicetos que crecen sobre Abies religiosa en el Eje Neovolcánico Transversal. Polibotánica 18: 33-51.

Vázquez del Mercado, M. 1977. Estudio sobre algunos Pezizales, principalmente de los estados de Veracruz y Oaxaca. Tesis licenciatura. Escuela Nacional de Ciencias Biológicas, Instituto Politécnico Nacional. México, D.F., México. $63 \mathrm{pp}$.

Welden, L. A., L. Dávalos y G. Guzmán. 1979. Segunda lista de hongos, líquenes y mixomicetos de las regiones de Uxpanapa, Coatzacoalcos, Los Tuxtlas, Papaloapan y Xalapa (México). Boletín de la Sociedad Mexicana de Micología 13: $151-161$.

Zhuang, W. Y. 2005. Re-disposition of specimens filed under Lachnea in HMAS. Fungal Diversity 18: 211-224. 